\section{報文}

(3)

\title{
充填層の有勃熱伝道 度*
}

杉山幸 男林・藤 津 正 則**

\section{[I] 緒 言}

充填層の有効熱云導度に関する研究は Kling ${ }^{10)}$ に始 まり非常に多くの報告がなされている2,3,4。すなおち流 れをともな弓場合の有効熱层導度については Wilhelm ${ }^{16)}$ の基本微分方程式による解析，Ranz ${ }^{111}$ による流れと直 角方向への流体混合に関する研究あるいは Argo \& Smith" らの理論式などがある。一方流れをとすなわな (場合については Schumann \& Voss, 6,8) らが半理論 式をだしている。また本邦においても八田・前田・川添

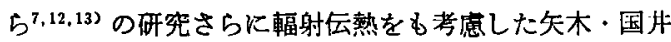
ら11,19.20)の理論式がある。しかしながらこれらの矼究は いすれれも充填層粒子の層数が充填層半径方向に非常に多 くある場合について検討されたるので，㬝数少ない場合 をも考虑に入れた理論解析は未だなされていない。また これら解析結果と実験結果との比較検討る比較的高温の 場合はあまり行なわれてはいない。

かかる見地に立って，筆者らは流れをともなわない充

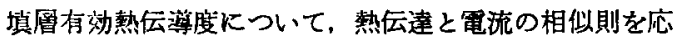
用し䅉数少い場合をる考虑に入れた理論解析を行ない， さらに高温に淤るる種々の実験結果と照合し式の検討を 行った。

\section{[II] 式の誘 草}

流れをともなわない充填層内の伝熱機構を，

i）固体内の伝導 (1)

ii）舜接する固体の接触面を通しての伝熱 (2)

iii）充填層内空間にあるガスの対流伝熱

iv）隣接固体間輻射伝熱

v）空間を通しての 1 凮とびの固体・固体 間輻射伝熱

からなっていると考える（2 層とびあるいはそれ以上の 固体・固体間輻射云熱は一心無視する)。

この伝熱機構を模型化して示せば Fig. 1 のよらであ る。

ここで熱伝澾と電流の相似則から，

$$
\left.\begin{array}{l}
q=k(\Delta T / \Delta x) S \\
i=E / R
\end{array}\right\}
$$

より $\Delta x / k S$ を抵抗と考える。

* 昭和 34 年 3 月 25 日

** 名古屋大学工学部化学工学科
同様にして輻射では $\sigma T^{3} \Delta T S$ から $1 / \sigma T^{3} S$ を抵抗と する。

いま Fig. 2 において

$R_{1}$ ：粒子内云導抵抗， $R_{2}$ ：固体接触抵抗，ガスの対 流伝熱抵抗括よび固体固体間輻射射云熱抵抗にもとつく 全抵抗， $R_{3}$ : 空隙を通して1層とび輻射层熱抵抗とす る。

まず半径方向に充填粒子が1 ケすなわち層数 】の場合 は, Fig. 3 より。

$$
R=\frac{1}{\frac{1}{R_{1}+2 R_{2}}+\frac{1}{R_{3}}}=\frac{R_{3}\left(R_{1}+2 R_{2}\right)}{R_{1}+R_{3}+2 R_{2}}
$$

となる。

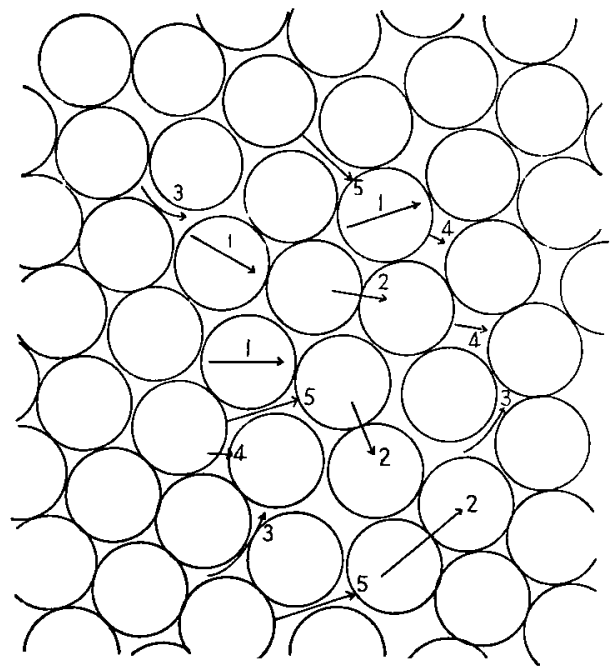

(1) heat transfer through solid

(2) heat transfer through the contact surface of soli 5

(8) heat transfer by convection of fluid

(4) heat transfer by radiation from packing to packing

(5) heat transfer by radiation from packing to packing beyond a packing

Fig. 1 A model of heat transfer in packed bed

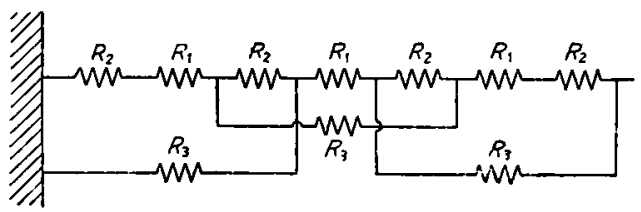

Fig. 2 A model of thermal resistance in packed bef 


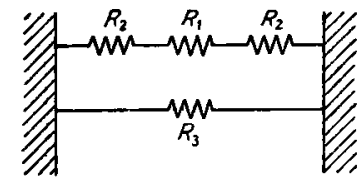

Fig. 3 A model of thermal resistance in one packed layer

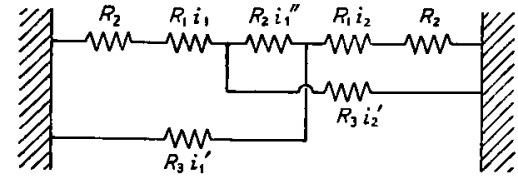

Fig. 4 A model of thermal resistance in two packed layers
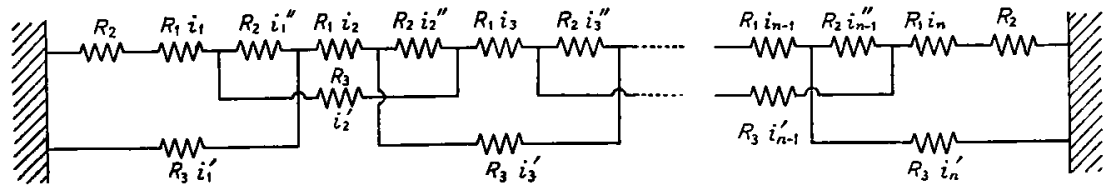

Fig. 5 A model of thermal resistance in $n$ packed layers

また層数 2 の場合は, Fig. 4より，

$$
\left.\begin{array}{l}
i_{1}+i_{1}{ }^{\prime}=i_{1}{ }^{\prime}+i_{1}{ }^{\prime}+i_{2}{ }^{\prime}=i_{2}+i_{2}{ }^{\prime}=i \\
i\left(R_{2}+R_{1}\right)+i_{1}{ }^{\prime \prime} R_{2}=i_{1}{ }^{\prime} R_{3} \\
i_{1}{ }^{\prime} R_{2}+\left(R_{1}+R_{2}\right) i_{2}=i_{2}{ }^{\prime} R_{3} \\
E=\left(R_{1}+R_{2}\right) i_{1}+i_{1}{ }^{\prime \prime} R_{2}+\left(R_{1}+R_{2}\right) i_{2}
\end{array}\right\}
$$

よって,

$$
\frac{E}{i}=R=\frac{R_{1} R_{2}+R_{2}+2 R_{1} R_{3}+3 R_{2} R_{3}}{R_{1}+3 R_{2}+R_{3}}
$$

となる。

一般に $n$ 層ならば, Fig. 5 より,

$$
i=i_{1}+i_{1}{ }^{\prime}=i_{2}+i_{2}{ }^{\prime}=i_{3}+i_{3}{ }^{\prime}=\cdots \cdots=i_{n}+i_{n}{ }^{\prime}
$$$$
=i_{1}{ }^{\prime \prime}+i_{1}{ }^{\prime}+i_{2}{ }^{\prime}=i_{2}{ }^{\prime \prime}+i_{2}{ }^{\prime}+i_{3}{ }^{\prime}=\cdots \cdots=i_{n-1}{ }^{\prime \prime}+i_{n-1}{ }^{\prime}+i_{n}{ }^{\prime}
$$
また，

$$
\begin{aligned}
& i_{1}\left(R_{1}+R_{2}\right)+i_{1}{ }^{\prime \prime} R_{2}=i_{1}{ }^{\prime} R_{3} \\
& i_{1}{ }^{\prime \prime} R_{2}+i_{2} R_{1}+i_{2}{ }^{\prime \prime} R_{2}=i_{2}{ }^{\prime} R_{3} \\
& i_{n-1}{ }^{\prime \prime} R_{2}+i_{n}\left(R_{1}+R_{2}\right)=i_{n}^{\prime} R_{3}
\end{aligned}
$$

よって

$$
\left.\begin{array}{l}
i_{1}\left(R_{1}+2 R_{2}+R_{3}\right)+i_{2} R_{2}=\left(R_{2}+R_{3}\right) i \\
i_{1} R_{2}+i_{2}\left(R_{1}+2 R_{2}+R_{3}\right)+i_{3} R_{2}=\left(2 R_{2}+R_{3}\right) i \\
\cdots \cdots \cdots \cdots \cdots \cdots \cdots \cdots \cdots \cdots \cdots \cdots \cdots \cdots \cdots \cdots \cdots \cdots \cdots \cdots \cdots \cdots \cdots \cdots \cdots \cdots \cdots \cdots \cdots \cdots \cdots \cdots \cdots \cdots \cdots \cdots \cdots \cdots \cdots \cdots \cdots \\
i_{n-1} R_{2}+i_{n}\left(R_{1}+2 R_{2}+R_{3}\right)=\left(R_{2}+R_{3}\right) i
\end{array}\right\}
$$

となり，この解を求めればよい。

一般に,

$i_{n-1} R_{2}+\left(R_{1}+2 R_{2}+R_{3}\right) i_{n}+R_{2} i_{n+1}=\left(2 R_{2}+R_{3}\right) i$

の解は,

$$
R_{2} a^{2}+\left(R_{1}+2 R_{2}+R_{3}\right) \alpha+R_{2}=0
$$

の根を $\alpha_{1}, \alpha_{2}$ とし，

$$
\begin{aligned}
& \left.\begin{array}{c}
\alpha_{1} \\
\alpha_{2}
\end{array}\right)=-\frac{R_{1}+2 R_{2}+R_{3}}{2 R_{2}} \pm \sqrt{\left(\frac{R_{1}+2 R_{2}+R_{3}}{2 R_{2}}\right)^{2}-1} \\
& i_{n}=C_{2} \alpha_{1}{ }^{n}+C_{2 \alpha_{2}}{ }^{n}+\frac{2 R_{2}+R_{3}}{R_{1}+2 R_{2}+R_{3}} i
\end{aligned}
$$

で与えられる。
$C_{1}, C_{2}$ は常数でこれを決めるため（4）式の第 1 を代 入すると，

$$
\left.\begin{array}{l}
C_{1}=\frac{R_{1}+2 R_{2}}{R_{1}+4 R_{2}+R_{3}} \quad \frac{\alpha_{2}{ }^{n+1}-1}{\alpha_{2}{ }^{n+1}-\alpha_{1}{ }^{n+1}} i \\
C_{2}=\frac{R_{1}+2 R_{2}}{R_{1}+4 R_{2}+R_{3}} \frac{1-\alpha_{1}{ }^{n+1}}{\alpha_{2}{ }^{n+1}-\alpha_{1}{ }^{n+1} l}
\end{array}\right\}
$$

となる。一方企電王 $E$ は,

$$
\begin{aligned}
i R= & E=i_{1}\left(R_{1}+R_{2}\right)+i_{1}{ }^{\prime \prime} R_{2}+i_{2} R_{1}+i_{2}{ }^{\prime \prime} R_{2}+\cdots \cdots \\
& +i_{n-1}{ }^{\prime \prime} R_{2}+i_{n}\left(R_{1}+R_{2}\right) \\
= & \left(i_{1}+i_{2}+\cdots \cdots+i_{n}\right)\left(R_{1}+2 R_{2}\right)-i R_{2}(n-1) \\
= & \left(R_{1}+2 R_{2}\right)\left\{C_{1} \frac{\alpha_{1}\left(1-\alpha_{1}{ }^{n}\right)}{1-\alpha_{1}}+C_{2} \frac{\alpha_{2}\left(1-\alpha_{2}{ }^{n}\right)}{1-\alpha_{2}}\right\} i \\
& +\left\{\frac{\left(R_{1}+2 R_{2}\right)\left(2 R_{2}+R_{3}\right)}{R_{1}+R_{3}+4 R_{2}} n-(n-1) R_{2}\right\} i
\end{aligned}
$$

これに $C_{1}, C_{2}$ の值を入れとると，

$$
\begin{aligned}
R= & \frac{2 R_{2}\left(R_{1}+2 R_{2}\right)^{2}}{\left(R_{1}+4 R_{2}+R_{3}\right)^{2}} \frac{\alpha_{2}-\alpha_{1}+\alpha_{2}{ }^{n}-\alpha_{1}{ }^{n}-\alpha_{2}{ }^{n+1}+\alpha_{1}{ }^{n+1}}{\alpha_{2}{ }^{n+1}-\alpha_{1}{ }^{n+1}} \\
& +\frac{\left(R_{1}+2 R_{2}\right)\left(2 R_{2}+R_{3}\right)}{R_{1}+4 R_{2}+R_{3}} n-(n-1) R_{2}
\end{aligned}
$$

となる。さて (1)，(3)，(6) 式に $R_{1}, R_{2}, R_{3}$ の值をそ れぞれ代入して $R=n l / k, S$ として $k_{e}$ すなわち有効熱伝 導度をるとめることができる。これから層数变化による 有効熱伝導度の值を知りらる。

\section{［III ] 式の模討ならびに応用}

上式より見て判るように $R_{1}, R_{2}, R_{3}$ などがわかれば有 効熱伝尊度をるとめ5るが，それらの抵抗は Fig. 6 か らむわかるよ5に，

$$
\begin{aligned}
& R_{1}=\frac{l_{s}}{k_{s} S_{s}}, \quad R_{2}{ }^{\prime}=\frac{l_{s}{ }^{\prime}}{k_{\mathrm{g}}{ }^{\prime} S_{\mathrm{g}}{ }^{\prime}}, \quad R_{2}{ }^{\prime \prime}=\frac{l_{\mathrm{r}}}{k_{\mathrm{g}} S_{\mathrm{v}}{ }^{\prime}}, \\
& R_{2}{ }^{\prime \prime \prime}=\frac{1}{\sigma^{\prime} T^{3} S_{v}}, \quad R_{3}=\frac{1}{\sigma^{\prime \prime} T^{3} S_{v}{ }^{\prime}}, \\
& \frac{1}{R_{2}}=\frac{k_{z}{ }^{\prime} S_{s}^{\prime}}{l_{s}^{\prime}}+\frac{k_{g} S_{v}}{l_{v}}+\sigma^{\prime} T^{3} S_{v .} \quad R=\frac{n l}{k_{\theta} S}
\end{aligned}
$$




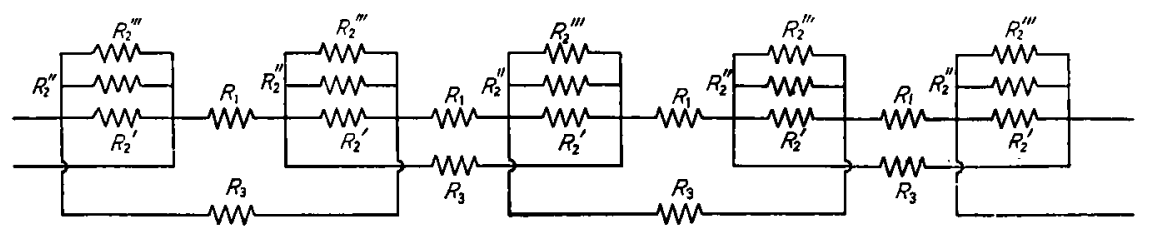

Fig. 6 A model of thermal resistance in packed bed

となる。ここで， $l_{\mathrm{a}}, S_{\mathrm{a}}, S_{\mathrm{s}}{ }^{\prime}, \cdots \cdots$ などの值や考方方につ いては議諭の余地があるが一応諸家の考え方9, 18, 19, 20) に ならって,

$$
\begin{aligned}
& l=l_{p}=\beta D_{p}, \quad l_{q}=\gamma D_{p}, \quad l_{q}^{\prime}=\tau^{\prime} D_{p}, \quad l_{v}=\varphi D_{p}, \\
& S_{\imath}=\left(1-\varepsilon^{1.3}\right) S, \quad S_{q}^{\prime}=\delta S, \quad S_{v}=\left(1-\varepsilon^{1.3}-\delta\right) S, \\
& S_{v}{ }^{\prime}=\varepsilon^{1.3} S
\end{aligned}
$$

とおくことにする。
いま $n$ が充分大きい場合について考点て見ると，その 場合は 6 式の第 1 項は消之て,

$$
\begin{aligned}
\frac{R}{n} & =\frac{\left(R_{1}+2 R_{2}\right)\left(2 R_{2}+R_{3}\right)}{R_{1}+4 R_{2}+R_{3}}-\frac{(n-1)}{n} R_{2} \\
& =\frac{R_{1} R_{3}+R_{1} R_{2}+R_{2} R_{3}}{R_{1}+4 R_{2}+R_{3}}
\end{aligned}
$$

となる。

これより，

よって,

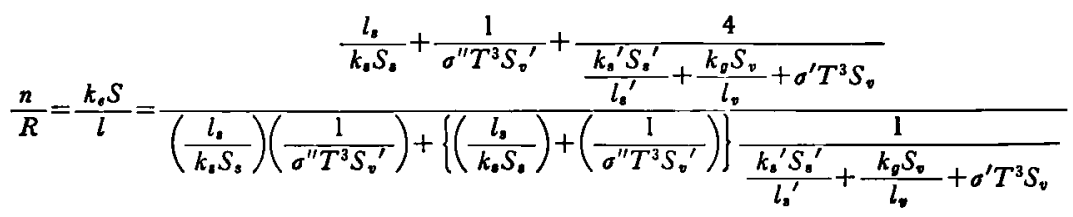

したがって,

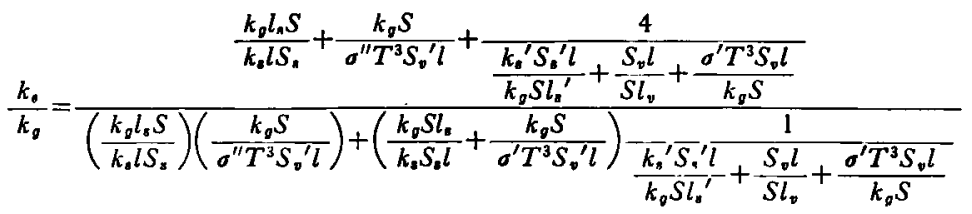

$$
\begin{aligned}
& \frac{k_{g}}{k_{s}} \frac{\gamma}{\left(1-\varepsilon^{1.3}\right) \beta}+\frac{k_{g}}{\sigma^{\prime \prime} T^{3} \varepsilon^{1.3} \beta D_{p}}+\frac{4}{\frac{k_{g}^{\prime}}{k_{g}} \frac{\delta \beta}{\gamma^{\prime}}+\frac{\left(1-\varepsilon^{1.3}-\delta\right) \beta}{\varphi}+\frac{\sigma^{\prime} T^{3}}{k_{g}}\left(1-\varepsilon^{1.3}-\delta\right)_{\beta} D_{p}}
\end{aligned}
$$

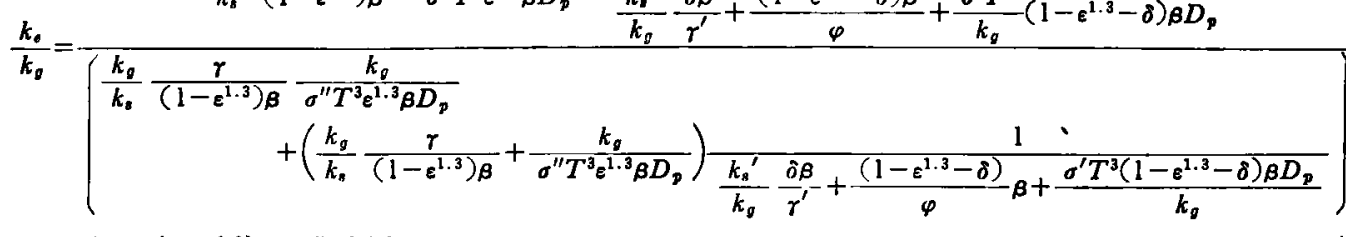

$$
\begin{aligned}
& \left(\frac{4 k_{s}\left(1-e^{1.3}\right)_{B}}{k_{g} r} \frac{\sigma^{\prime \prime} T^{3} \varepsilon^{1.3} \beta D_{p}}{k_{g}}\right. \\
& \left.+\left(\frac{k_{s}}{k_{g}} \frac{\left(1-\varepsilon^{1.3}\right) \beta}{r}+\frac{\sigma^{\prime \prime} T^{3} \varepsilon^{1.3} \beta D_{p}}{k_{g}}\right)\left(\frac{k_{s}^{\prime}}{k_{g}} \frac{\delta \beta}{\gamma^{\prime}}+\frac{\left(1-\varepsilon^{1.3}-\delta\right)}{\varphi} \beta+\frac{\sigma^{\prime} T^{3}\left(1-\varepsilon^{1.3}-\delta\right) \beta D_{p}}{k_{p}}\right)\right) \\
& \frac{k_{s}}{k_{g}} \frac{\left(1-\varepsilon^{1.3}\right) \beta}{\gamma}+\frac{\sigma^{\prime \prime} T^{3} \varepsilon^{1.3} \beta D_{p}}{k_{g}}+\frac{k_{g}^{\prime}}{k_{g}} \frac{\delta \beta}{\gamma^{\prime}}+\frac{\left(1-e^{1.3}-\delta\right)}{\varphi} \beta+\frac{\sigma^{\prime} T^{3}}{k_{g}}\left(1-\varepsilon^{1.3}-\delta\right)_{\beta} D_{\text {, }}
\end{aligned}
$$

さて一応 $\beta=1, \gamma=1, \delta=0^{19,20)}$ とすると，

$$
\frac{\frac{4 k_{g}\left(1-\varepsilon^{1.3}\right)}{k_{g}} \frac{\left(\sigma^{\prime \prime} T^{3} e^{1.3} D_{p}\right)}{k_{g}}+\left\{\frac{k_{g}}{k_{g}}\left(1-e^{1.3}\right)+\frac{\sigma^{\prime \prime} T^{3} e^{1.3} D_{p}}{k_{g}}\right\}\left\{\frac{\left(1-\varepsilon^{1.3}\right)}{\varphi}+\frac{\sigma^{\prime} T^{3}\left(1-\varepsilon^{1.3}\right) D_{p}}{k_{g}}\right\}}{\frac{k_{g}}{k_{g}}\left(1-\varepsilon^{1.3}\right)+\frac{\sigma^{\prime \prime} T^{3} e^{1.3} D_{p}}{k_{g}}+\frac{1-\varepsilon^{1.3}}{\varphi}+\frac{\sigma^{\prime} T^{3}\left(1-\varepsilon^{1.3}\right) D_{p}}{k_{g}}}
$$

あるいは,

$$
\frac{k_{q}}{k_{q}}=\frac{1}{\frac{1}{\frac{k_{q}}{k_{q}}\left(1-\varepsilon^{1.3}\right)+\frac{\sigma^{11} T^{3} e^{1.3} D_{p}}{k_{g}}}+\frac{1}{\frac{1-\varepsilon^{1.3}}{\varphi}+\frac{\sigma^{\prime} T^{3}\left(1-e^{1.3}\right)}{k_{g}} D_{p}}}
$$




$$
\begin{aligned}
& +\frac{\frac{k_{q}}{k_{q}}\left(1-\varepsilon^{1.3}\right) \frac{\sigma^{\prime \prime} T^{3} \varepsilon^{1.3} D_{p}}{k_{g}}}{\frac{k_{q}}{k_{g}}\left(1-\varepsilon^{1.3}\right)+\frac{\sigma^{\prime \prime} T^{3} \varepsilon^{1.3} D_{p}}{k_{q}}+\frac{1-\varepsilon^{1.3}}{\varphi}+\frac{\sigma^{\prime} T^{3}}{k_{q}}\left(1-\varepsilon^{1.3}\right) D_{p}} \\
& =\frac{\left(1-\varepsilon^{1.3}\right)}{\left(\frac{k_{q}}{k_{q}}\right) \frac{1}{1+\left(\frac{\sigma^{11} T^{3} \varepsilon^{1.3} D_{p}}{k_{q}}\right)\left(\frac{k_{q}}{k_{g}\left(1-\varepsilon^{1.3}\right)}\right)}+\frac{1}{\frac{1}{\varphi}+\frac{\sigma^{\prime} T^{3}}{k_{g}} D_{p}}} \\
& +\frac{\varepsilon^{1.3} \sigma^{\prime \prime} T^{3} D_{p}}{\frac{1}{4}+\left\{\frac{\sigma^{\prime \prime} T^{3} \varepsilon^{1.3} D_{p}}{k_{g}\left(1-\varepsilon^{1.3}\right)}+\frac{1}{\varphi}+\frac{\sigma^{\prime} T^{3} D_{p}}{k_{q}}\right\} \frac{k_{g}}{4 k_{q}}}
\end{aligned}
$$

となる。

（8）式が充填層粒子の層数が充分多い場合の一般式で ある。液充澫の場合は輻射の項をはぶいて,

となる。

$$
\frac{k_{s}}{k_{q}}=\frac{1-\varepsilon^{1.3}}{\frac{k_{0}}{k_{s}}+\varphi}
$$

なお， $\sigma^{\prime}, \sigma^{\prime \prime}$ の値は矢木らにならって,

$$
\begin{aligned}
& \sigma^{\prime} T^{3}=0.1952\left(\frac{p}{2-p}\right)\left(\frac{t+273}{100}\right)^{3} \\
& \pi^{\prime \prime} T^{3}=0.1952 \frac{1}{1+\frac{\varepsilon}{2(1-\varepsilon)} \cdot \frac{1-p}{p}}\left(\frac{t+273}{100}\right)^{3}
\end{aligned}
$$

を用いてよかろ5。甲については，談家の実測值 ${ }^{17)}$ （8）または（9）式に代入して $\varphi$ を計算しこれと との 関係を Fig. 7 に示した。計算にあたっては Fig. 7 よ り

さて上式の誘導にあたっては，空隙・空㗂の輻射伝熱 は次に固体を通しての伝熱となると考光たるのですすな わち固体伝導抵抗が常化考虑されているすのであり，こ の点矢木ら ${ }^{192,20)}$ の考方方とややことなっている。した がって輻射抵抗と固体伝導抵抗が充分差異があり，かつ 空隙・空隙の輻射伝熱が有効熱伝導度に充分影留を与之

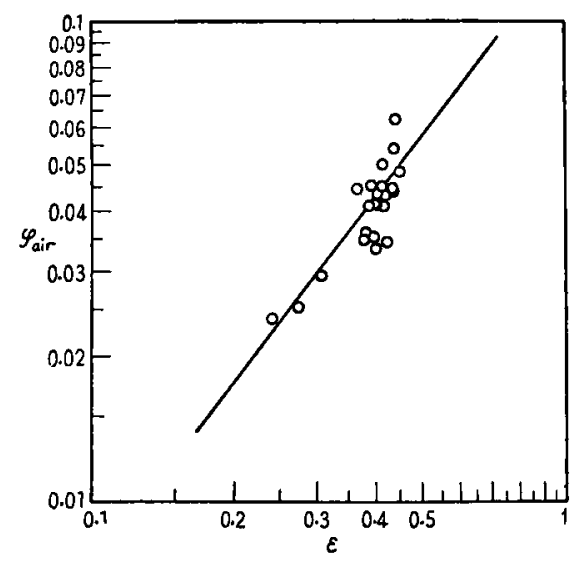

Fig. 7 Value $\varphi$ calculated from previously reported data" ${ }^{\text {I' }}$
る場合は両者の式から計算した值は開きがでてくる。 さて (8) 式は計算にあたってやや面倒であるので，い ま(7) 式,

$$
\frac{R}{n}=\frac{R_{1} R_{3}+R_{2} R_{3}+R_{1} R_{2}}{R_{1}+4 R_{2}+R_{3}}
$$

において，

$$
R_{2} / R_{1}=m, \quad R_{3} / R_{2}=h
$$

と䋆ば。

$$
R_{3}=m h R_{1}
$$

となり，したがって、

$$
\begin{aligned}
& \frac{R}{n}=\frac{h R_{1}+m h R_{1}+R_{1}}{\frac{1}{m}+4+h} \\
& \frac{R}{n R_{1}}=\frac{m(h+1+m h)}{4 m+m h+1}
\end{aligned}
$$

となる。

$$
\begin{aligned}
& \text { ここで, } \\
& \begin{aligned}
\frac{R}{n R_{1}}= & \frac{l}{k_{\theta} S} \frac{k_{s} S_{\mathrm{s}}}{l_{\mathrm{s}}}=\frac{D_{p}}{k_{s} S} \cdot \frac{k_{s}\left(1-\varepsilon^{1.3}\right) S}{D_{p}} \\
= & \frac{k_{s}}{k_{\theta}}\left(1-\varepsilon^{1.3}\right) \\
\frac{R_{2}}{R_{1}}=m= & \frac{k_{s}\left(1-\varepsilon^{1.3}\right) S}{D_{p}} \\
& \cdot \frac{1}{\left\{\frac{k_{g}}{D_{p}}\left(1-\varepsilon^{1.3}\right)+\sigma^{\prime} T^{3}\left(1-\varepsilon^{1.3}\right)\right\} S} \\
= & \frac{k_{g}}{k_{g}} \frac{1}{\frac{1}{\varphi}+\frac{\sigma^{\prime}}{T^{3} D_{p}}}
\end{aligned}
\end{aligned}
$$

すなわち，

$$
m \frac{k_{q}}{k_{s}}=\frac{1}{\frac{1}{\varphi}+\frac{\sigma^{\prime}}{T^{3} D_{p}}}
$$

となる。

また。

$$
\begin{aligned}
& \frac{R_{a}}{R_{2}}=h=\frac{1}{\sigma^{\prime \prime} T^{3} \varepsilon^{1.3} S}\left\{\frac{k_{g}}{\varphi D_{p}}\left(1-\varepsilon^{1.3}\right)+\sigma^{\prime} T^{3}\left(1-\varepsilon^{1.3}\right)\right\} S \\
& \text { ここで近似的に } \sigma^{\prime \prime}=\sigma^{\prime} \text { とおくならば } \\
&=\frac{\left(1-\varepsilon^{1.3}\right)}{\varepsilon^{1.3}}\left[-\frac{1}{\left(\frac{\sigma^{\prime} T^{3} D_{p}}{k_{q}}\right)(\varphi)}+1\right]
\end{aligned}
$$

(15) 


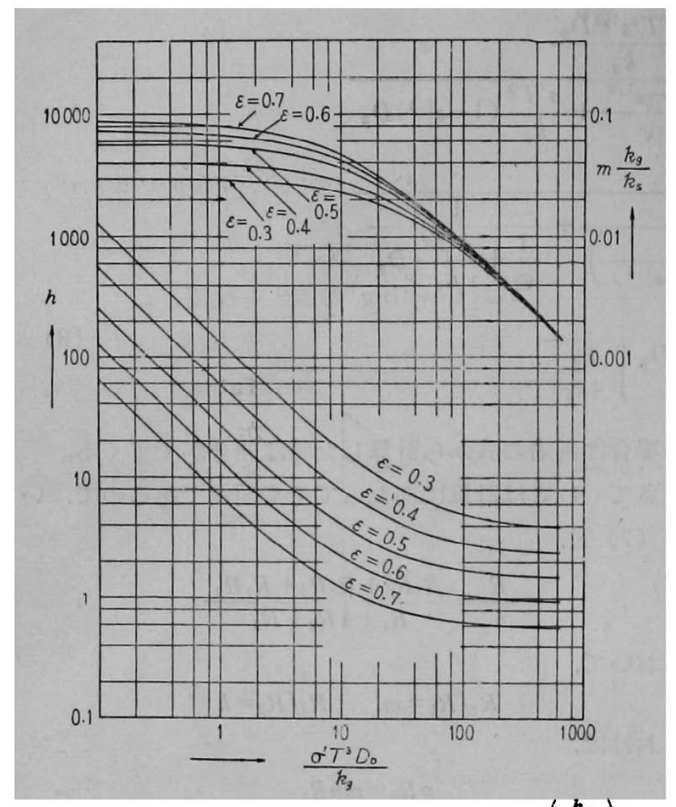

Fig. 8 Relation between $R_{3} / R_{2},\left(R_{2} / R_{1}\right)\left(\frac{k_{g}}{k_{q}}\right)$ and $\varepsilon, \frac{\sigma^{\prime} T^{3} D_{p}}{R_{g}}$

となる。よって $m, h$ をと, $\frac{\sigma^{\prime} T^{3} D_{0}}{k_{g}}$ のバラィータで図 示しておけば有効熱伝導率は近似的ながら非常に容易に むとめることができる。このようにして図示したのが Fig. 8 である。

層数少ない埸合む同様にもとめらるが，たとえば， $R_{2}=R_{3}$ としたとき， $R_{2} / R_{1}=m$ として，

$$
\begin{array}{rlrl}
n=1 ; & \frac{R}{n} & =R_{1}\left\{\frac{m(1+2 m)}{(1+3 m)}\right\} \\
n=2 ; & & =R_{1}\left\{\frac{m(3+4 m)}{(1+4 m)}\right\} \times \frac{1}{2} \\
n=3 ; & =R_{1} \frac{m\left(8 m^{2}+13 m+5\right)}{7 m^{2}+6 m+1} \times \frac{1}{3} \\
n=\infty ; & =R_{1} \frac{m(2+m)}{1+5 m}
\end{array}
$$

となる。したがって，

\begin{tabular}{rrrrr}
\multicolumn{5}{c}{$(R / n) / R_{1}$} \\
& $m=10$ & $m=1$ & $m=0.1$ & $m=0.01$ \\
$n=1$ & 6.78 & $0.7 \overline{5}$ & $0.092 \overline{5}$ & 0.0099 \\
$=2$ & $5.2 \overline{5}$ & 0.70 & 0.122 & $0.014 \overline{5}$ \\
$=3$ & 4.10 & 0.62 & 0.127 & 0.0161 \\
$=\infty$ & $2.3 \overline{5}$ & 0.50 & 0.147 & 0.0192
\end{tabular}

となる。これよりわかるように平均周当り抵抗したがっ

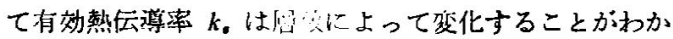
る。

[IV] 実雊結果ならひに考察

ここで $n$ が充分大きな場台について行なった买験結果

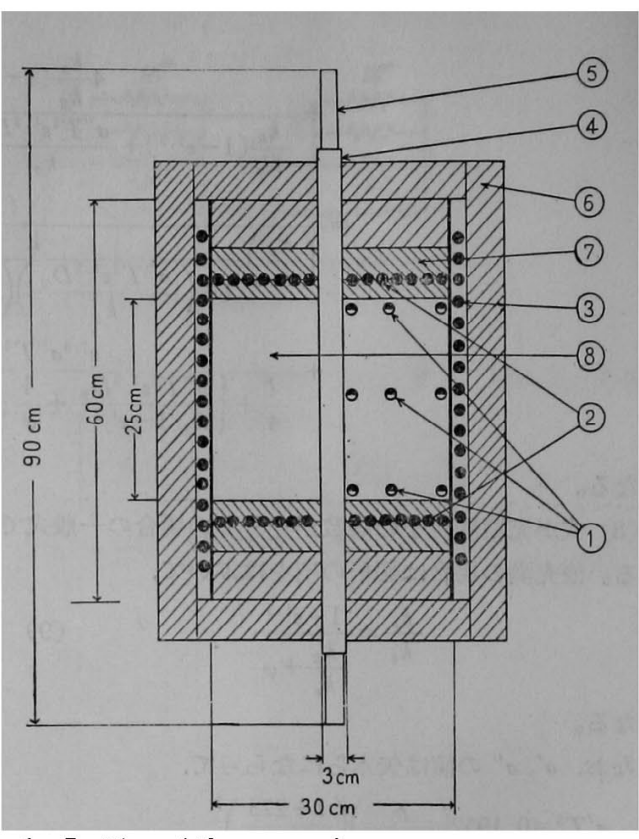

1. Position of thermocouples

2. Nichrome wire employed to counterbalance heat loss

3. Nichrome were employed to counterbalance heat loss through wall

4. Siliea tube

5. $\mathrm{SiC}$ electric heater

6. Refractory brick

7. Insulating fire brick

8. Packed bed

Fig. 9 Experimental apparatus (a) used to measure effective thermal conductivity

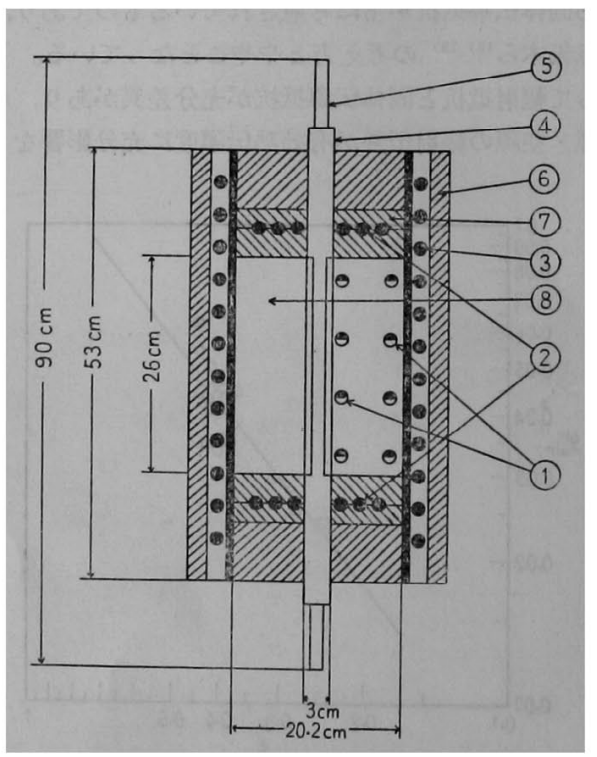

Fig. 10 Experimental apparatus (b) used to measure effective thermal conductivity 
をしめす。使用した装置が Fig. 9, 10 に示されてある。 充填物粒子の大きさにより適宜 2 つの装置を併用した。 䓡源には $\mathrm{SiC}$ 発熱体を用い一定時間ことに所定の電力 になるように調節した。塔の上部および下部にニクロム

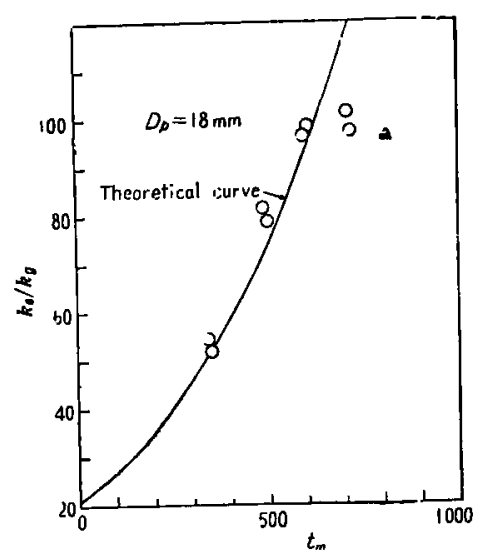

Fig. 11 Data on iron spheres

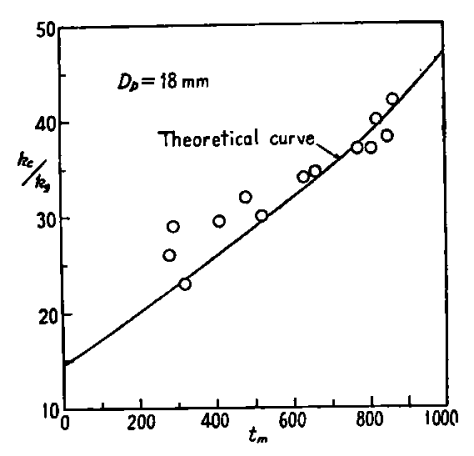

Fig. 12 Data on porcelains

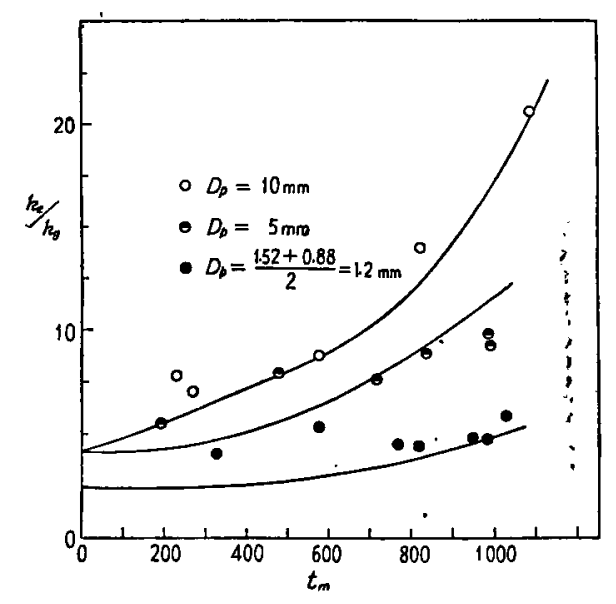

Fig. 15 Data on insulating fire bricks

線加熱源をおき垂直方向の熱の流れを防いだ。また装置 の外壁にも二クロム線を入れ熱損失を防ぐとともに所定 の温度差煌つよう調節した。温度测度はフルメル・ク ロメル線を用いとくに高温の場合は白金・白金ロジウム 線を使用した。些験值と(8)式よりもとめた計算値との 比較を Fig. 11，12，13，14，15 に示した。空気の熱云

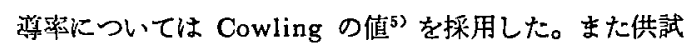

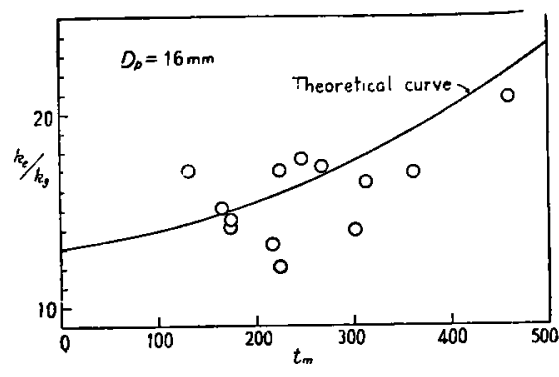

Fig. 13 Data on glass spheres

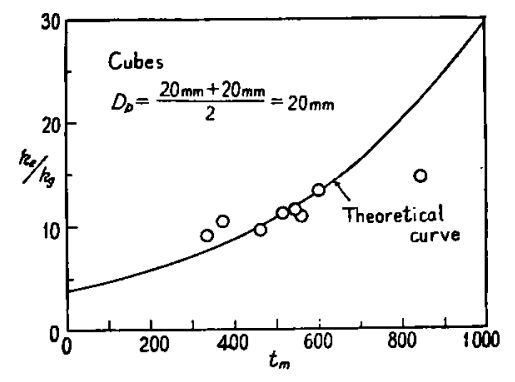

Fig. 14 Data on insulating fire bricks 充填物の特性值を Table 1 に示した。图 から判るように金属 球，磁製球の場合は実 跧値と計算値はよい一 致を示している。また 断熱耐火煉瓦 F 2 の場 合では粒径の比較的大 きな場合かなりよい一 致をみるが，粒径が小 さくなると両者にやや 開きがでる。これは固 体粒子自身の熱伝導率 の変化によるむのと考 えられる。

さらに比較的高温で 行なれれた矢杖の実 験について, 同氏らの 理諭式との計算の比較 の 1 例を Fig. 16 に示 した。

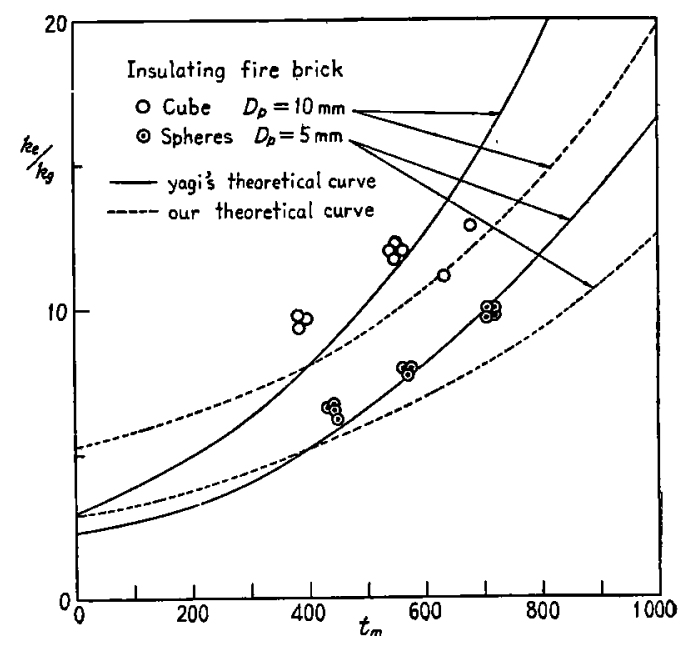

Fig. 16 Comparison between the values obtained of our theoretical equation with the experimental data previously reported ${ }^{20}$ 
Table 1 Characteristics of packings used in the experiments on effective thermal conductivity

\begin{tabular}{|c|c|c|c|}
\hline Packing & $\underset{[\mathrm{mm}]}{D_{p}}$ & $\varepsilon$ & $\begin{array}{c}K_{\mathrm{a}}[\mathrm{kcal} / \\
\left.\mathrm{m} \cdot \mathrm{hr} \cdot{ }^{\circ} \mathrm{C}\right]\end{array}$ \\
\hline Iron sphere & 18 & 0.43 & 45 \\
\hline Porcelain sphere & 18 & 0.43 & 1.4 \\
\hline Glass sphere & 16 & 0.435 & 1 \\
\hline Insulating fire brick sphere & 5 & 0.5 & 0.18 \\
\hline " & $1.2^{*}$ & 0.7 & 0.18 \\
\hline Insulating fire brick cube & 20 & 0.43 & 0.18 \\
\hline * mean value & 10 & 0.43 & 0.18 \\
\hline
\end{tabular}

\section{[V] 括}

筆者らは就伝達と電流の相似則を沁用して，流れをと もなわない充填層有効熱层導度について，熱の流れ方向 の充填層粒子数が少い場合をも考虏に入れた新らしい理 論式を誘導した。その結果, 充填層半径方向の粒子数が 6〜7 以上でほぼ定常になることが認められた。さらに 誘導された一般式の計算の複雑さを省くため， $R_{3} / R_{2}$, $R_{2} / R_{1}, \& ， \frac{\sigma^{\prime} T^{3} D_{q}}{k_{q}}$ の 4 この䑯次元項の関保を chart にし計算が極めて容易に行なわれるよう考虑した。層数 の充分大なる場合について行った実験結果は理論式とよ い一致をしめした。

付記 本研究を行らに当り実験ならびに計算の一部を 手伀って頂いた平田仁宏氏に謝意を表す。

\section{Nomenelature}

$C_{1}, C_{2}$ : constants given by Eq. (5)

$D_{p}$ : average diameter of packings

$E$ : voltage

[volt]

$h$ : ratio of $R_{3}$ to $R_{2}$

$i$ : electric current [ampere]

$i_{n}$ : electric current passed through $R_{1}$ [ampere]

$i_{n}{ }^{\prime}$ : electric current passed through $R_{3}$ [ampere]

$i_{n}{ }^{\prime \prime}$ : electric current passed through $R_{2}$ [ampere]

$k_{\varepsilon}$ : effective thermal conductivity in packed bed

$$
\left[\mathrm{kcal} / \mathrm{m} \cdot \mathrm{hr} \cdot{ }^{\circ} \mathrm{C}\right]
$$

$k_{g}$ : thermal conductivity of fluid

$$
\left[\mathrm{kcal} / \mathrm{m} \cdot \mathrm{hr} \cdot{ }^{\circ} \mathrm{C}\right]
$$

$k_{\text {a }}$ : thermal conductivity of packing

$$
\left[\mathrm{kcal} / \mathrm{m} \cdot \mathrm{hr} \cdot{ }^{\circ} \mathrm{C}\right]
$$

$l$ : average length between centers of packings

$l_{r}$ : effective length between centers of packings

$l_{3}$ : effective length for heat flow by conduction in packing

$l_{s}{ }^{\prime}$ : effective length relating to heat flow by conduction of contact surface [m]

$l_{v}$ : effective length for heat flow by convection of fluid $[\mathrm{m}]$

$m$ : ratio of $R_{2}$ to $R_{1}$ [-]

$n$ : numbers of packings in the direction of heat flow

$p$ : emissivity of packing

$q$ : heat flux

$[\mathrm{kcal} / \mathrm{hr}$ ]

$R$ : resistance :[ohm]

$R_{1}$ : resistance to conduction in packing [ohm]

$R_{2}$ : composite resistance of $R_{2}{ }^{\prime}, R_{2}{ }^{\prime \prime}$, and $R_{2}{ }^{\prime \prime \prime}$ in parallel

[ohm]

$R_{2}^{\prime}$ : contact resistance to conduction of [ocking ]

$\boldsymbol{R}_{2}{ }^{\prime \prime}$ : resistance to convection of fluid [ohm]

$R_{2}^{\prime \prime \prime}$ : resistance to radiation between adjacent packings

[ohm]

$R_{3}$ : resistance to radiation between packings beyond a packing

[ohm]

$S$ : area for heat flow $\left[\mathrm{m}^{2}\right]$

$S_{s}$ : area for heat flow by conduction in packing $\left[\mathrm{m}^{2}\right]$

$S_{s}^{\prime}$ contact area of packing $\quad\left[\mathrm{m}^{2}\right]$

$S_{v}$ : area for heat flow by convection of fluid $\left[\mathrm{m}^{2}\right]$

$S_{v}{ }^{\prime}$ : area for heat flow by radiation between packings beyond a packing $\left[\mathrm{m}^{2}\right]$

$T:$ temperature

$\Delta T:$ temperature difference

$t_{m}$. mean temperature in packed beds

$a_{1}, a_{2}$ : solution of Eq. (4)

$\beta:=l_{p} / D_{p}$

$[-]$

$r:=l_{\mathrm{s}} / D_{p}$

$[-]$

$\gamma^{\prime}:=l_{\mathrm{a}}^{\prime} / D_{\mathrm{p}}$

[-]

$\delta:=S_{\mathrm{a}}^{\prime} / S$

$\varepsilon$ : fractional void $[-]$

$\varphi:=l_{v} / D_{p}$ $[-]$

$\sigma T^{3}, \sigma^{\prime} T^{3}, \sigma^{\prime \prime} T^{3}$; heat transfer coefficient of radiation

$\left[\mathrm{kcal} / \mathrm{m}^{2} \cdot \mathrm{hr} \cdot{ }^{\circ} \mathrm{C}\right]$ 


\section{Literature cited}

1) Argo, W. E. and Smith, J. M. : Chem. Eng. Progress, 49, 443 (1953)

2) Bunnell, D. G. et al : Ind. Eng. Chem., 41, 1977 (1947)

3) Campbell, J. M. and Huntington, R. L.: Pet. Ref., 31, No. 2, 123 (1952)

4) Coberly, C. A. and Marshall, Jr. W. R. Chem. Eng. Progress, 47, 141 (1951)

5) Cowling, C.: "Mathematical Theory of Non-Uniform Gases" Cambridge University Press (1952)

6) Damköhler, G.: Z. Elektrochem., 42, 846 (1936) ; 43, 8 (1937)

7) Hatta, S. and Maeda, S. : Chem. Eng. (Japan), 13, 79 (1949)

8) Kanuluick, W. G. and Martin, L. H. - Proc. Royal Soc. (London), A 141, 144 (1933)

9) Kimura, M.: Chem. Eng. (Japan), 21, 472 (1957)
10) Kling, G. : Forsch. Geb. Ingenieurw, 9, 28 (1938)

11) Kunii, D. : Chem. Eng. (Japan), 20, 564 (1956)

12) Maeda, S. and Kawazoe, K. . ibid., 17, 276 (1953)

13) Maeda, S. and Kawazoe, K.: ibid., 18, 276 (1954)

14) Ranz, W. E. . Chem. Eng. Progress, 48, 247 (1952)

15) Schumann, J. E. W. and Voss, V. . Fuel, 13, 249 (1934)

16) Singer, E. and Wilhelm, R. H. . Chem. Eng. Progrcss, 46, $343(1950)$

17) Wilhelm, R. H., Johnson, W. C., Wynkoop, R, and Collier, D. W. . Chem. Eng. Progress, 44, 105 (1948)

18) Wyllie, M. R. J. and Gregory, A. R.: Trans. Am. Inst. Minning Met. Engrs., 198, 103 (1953)

19) Yagi, S. and Kunii, D. : Chem. Eng. (Japan), 18, 576 (1954)

20) Yagi, S. and Kunii, D.: A. I. Ch. E. Journal, 3, 373 (1957)

\title{
Effective Thermal Conductivities in Packed Beds
}

\author{
S. Sugiyama* \& M. Fujitsu*
}

Electric analogue circuits are used for evaluating the effective thermal conductivities in packed beds without fluid flow, taking into consideration the numbers of packings placed in the direction of heat flow.

In the analysis, it is assumed that the thermal resistance within packed beds may occur in the following forms :

(1) Thermal resistance of packing itself.

(2) Thermal resistance of contact area.

(3) Thermal resistance of fluid between packings.

(4) Thermal resistance to heat radiation from packing to packing.

(5) Thermal resistance to heat radiation from packing to adjoining packing beyond one.

The equations obtained are given in the form of Eqs. 1, 3, 6 and 7. Introducing the values of thermal resistance into Eq. 7, we have obtained Eq. 8 which expresses the relation of $k_{\varepsilon} / k_{g}$.

Experiments have been carried out with various packings: iron, porcelain, glass balls and insulating fire bricks at the temperature between 100 and $1000^{\circ} \mathrm{C}$.

Comparisons between the experimental results and the values obtained from Eq. 8 are shown in Figs. $11,12,13,14$ and 15. The calculation can be readily handled by employing the chart of Fig. 8, without using the complicated formula, Eq. 8. The difference between the value obtained from the chart (Fig. 8) and that obtained from Eq. 8 is small and negligible.

\footnotetext{
* Department of Chemical Engineering, INagoya University
} 